\title{
On the Capacity of Slotted Aloha Based Multi-Hop Virtual MIMO Systems
}

\author{
Chiara Buratti \\ WiLAB, DEIS \\ University of Bologna, Italy \\ Email: c.buratti@unibo.it
}

\author{
Alberto Zanella \\ WiLAB, IEIIT \\ National Research Council (CNR), Italy \\ Email: alberto.zanella@cnr.it
}

\author{
Roberto Verdone \\ WiLAB, DEIS \\ University of Bologna, Italy \\ Email: roberto.verdone@unibo.it
}

\begin{abstract}
The performance of multi-hop Virtual MultipleInput-Multiple-Output (V-MIMO) systems, when Slotted Aloha is used to select the radio resource at each hop, is investigated. Source, destination and relaying nodes, cooperate with neighboring devices (distributed according to a Poisson Point Process) to exploit spatial diversity by means of the concept of Virtual Antenna Array (VAA). Performance is evaluated in terms of outage probability, defined as the probability that the capacity between source and destination is smaller than a given threshold. It is computed as a function of the density of cooperating nodes, and the number of relaying VAAs. It is shown that depending on the level of required capacity, the optimum number of slots per frame in the slotted structure may differ. The performance degradation with Slotted Aloha with respect to a deterministic centralised re-use of radio resources, is assessed.
\end{abstract}

Index Terms-Virtual MIMO, Slotted Aloha, Capacity.

\section{INTRODUCTION}

Recently, wireless systems based on the concept of Virtual (or distributed) Multiple-Input-Multiple-Output (V-MIMO) have attracted the interest of the research community [1], [2]. In a V-MIMO system, nodes cooperate to create clusters, also denoted as Virtual Antenna Arrays (VAAs), which share their own resources (antennas, transceivers, energy, etc) for transmission and/or reception purposes [1]. VAAs are formed by nodes who are in close contact with the main ones that need to transfer information; the number of nodes in each VAA can be random, depending on their positions and the radio channel status. The performance of multi-hop cooperative strategies are investigated in [3], whereas in [4] a novel cooperative diversity system model for multi-hop scenarios is proposed and compared to conventional two-hop cooperative systems, under the condition that all terminals are equipped with only one antenna.

In most of the papers addressing a multi-hop V-MIMO scenario (i.e., [1], [5]), communication between VAAs is carried out using frequency or time division multiple access. In these scenarios, source-destination paths with few hops were investigated and the overall radio resource available was fractionated among the inter-VAA links. It is well known that the use of orthogonal radio resources (e.g., each link uses a different unitary resource) leads to interference-free links, but tends to decrease the overall system spectral efficiency. This performance degradation increases with the number of hops. On the contrary, in case of radio resource reuse, links are affected by interference, but a sufficient amount of radio resources can be assigned to each link also if the complete source-destination path is composed of many hops.

In [6] the impact of non-orthogonal reuse of resources in a six-hop V-MIMO system with perfect reuse, is investigated. In this paper, the results of [6] are extended, by (i) considering paths of any length and (ii) assuming resources are assigned to each hop independently, through a Slotted Aloha procedure: the time axis is split into frames, each composed of a given number of slots; assuming perfect synchronisation among nodes, each VAA in the multi-hop chain picks a random slot. This approach provides worse performance than the case of a centralised procedure implementing a perfect deterministic reuse of the time slots. On the other hand, it is simpler as independent resource assignments are made at each hop.

As in [5] (where reuse was absent), we assume that cooperating nodes are distributed according to a Poisson Point Process (PPP) and that nodes are equipped with both a longand a short-range communication system: the former can be used for inter-VAA communications and the latter for intraVAA networking. We define, as a performance metric, the probability that the ergodic capacity of the whole V-MIMO system is less than a given value (here denoted as outage probability). The behavior of the outage probability is shown by varying different system parameters, and comparing the cases of Slotted Aloha and deterministic assignment of radio resources. Numerical results show that depending on the level of required capacity, the optimum number of slots per frame in the slotted structure may differ.

\section{SySTEM DESCRIPTION}

Throughout the article vectors and matrices are indicated by bold, and $\mathbf{I}$ is the identity matrix. $\left\{a_{i, j}\right\}_{i, j=1, \ldots, M}$ is an $M \times M$ matrix with elements $a_{i, j}=\{\mathbf{A}\}_{i, j}, \dagger$ is the operator of conjugation and transposition. Also, $\mathbb{E}\{\cdot\}$ denotes expectation, $\mathbb{P}\{\mathcal{E}\}$ denotes the probability of the event $\mathcal{E}$ and $B(x, y)$ denotes an indicator function equal to one for $x<y$ and zero otherwise.

\section{A. Multi-hop Paths}

We consider a multi-hop V-MIMO system (see Figure 1), where a source node transmits data to a destination node via some relays. In the following, source, relays and destination 
will be denoted as main nodes and $Z$ will denote the number of hops. A number of ancillary nodes are located in non overlapped arbitrary areas $\mathcal{A}_{\mathrm{S}}, \mathcal{A}_{\mathrm{R}_{\mathrm{i}}}$ (where $i$ denotes the $i \frac{\text { th }}{}$ relay) and $\mathcal{A}_{\mathrm{D}}$, around the main nodes. In particular, ancillary nodes are distributed over the areas according to a PPP. With such model the probability of having one node in the infinitesimal area $\delta \mathcal{A}$ is $\eta / \delta \mathcal{A}$, where $\eta \prime$ denotes the nodes' density [8]. Owing to the possible presence of mechanisms such as sleeping schedules implemented at nodes, only a subset of the ancillary nodes are active simultaneously; we denote as $\eta$ the density of the active nodes. We assume that a decode and forward strategy is implemented at the $Z-1$ relays [1], and that the main nodes can cooperate only with the ancillary nodes which guarantee a feasible quality of the link.

The nodes that actually communicate with the main nodes are called cooperating nodes; we denote as $n_{\mathrm{S}}, n_{\mathrm{R}_{\mathrm{i}}}$ and $n_{\mathrm{D}}$ the total number of nodes (cooperating and main) at the source VAA, at the $r_{i}{ }^{\text {th }}$-relay VAA and at the destination VAA, respectively. We assume that each VAA is formed independently, and the set of cooperating nodes at a given transmission is independent from that at a following transmission, owing to the changing conditions of the radio channel, the node sleeping schedules, etc..

We also assume that the source-relay, relay-relay and relaydestination distances are much larger than the distance between a main node and its cooperating nodes. Therefore the short-range radio interface can be used only to transmit/receive data to/from the main node and its cooperating nodes (intraVAA networking). With this assumption, intra-VAA communication does not have any effect on the other VAAs. We also assume that all the VAAs have always data to be transmitted toward the next hop. The procedure of data forwarding from source to destination is described in [5].

The maximum number of cooperating nodes that the main nodes can actually handle is obviously limited by their hardware equipment: we denote this number as $M$.

\section{B. Slotted Aloha}

We assume that a Slotted Aloha protocol is used by the different VAAs to access the channel; the whole amount of radio resource available for inter-VAA communications is divided among $R$ unitary resources. In other words, each frame has $R$ time slots. Therefore, once a VAA has data to be transmitted, the main node of the VAA will select randomly one out of the $R$ available time slots in the next frame and will communicate the choice to its cooperating nodes. In this way, all nodes of a given VAA (cooperating nodes plus the main node) will simultaneously access the channel by using the same unitary resource, selected with probability $1 / R$. Given that time slot selection is made independently at all VAAs, depending on the value of $R$ there is a nonzero probability that each of the other links is using the same time slot and interference is present.

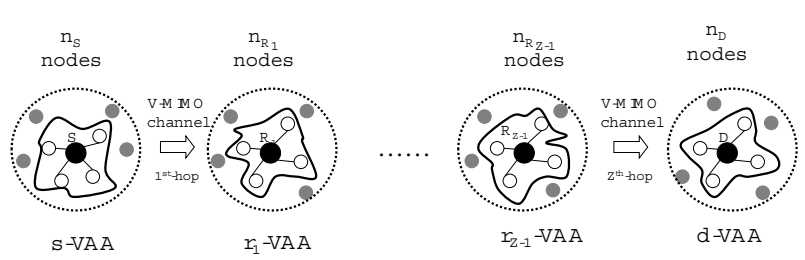

Fig. 1. The Virtual MIMO communication system.

\section{Propagation and Connectivity Models}

We assume that the ratio between the receive power, $P_{\mathrm{R}}$ and the transmit power, $P_{\mathrm{T}}$, is $\frac{P_{\mathrm{R}}}{P_{\mathrm{T}}}=\frac{d^{-\beta} \cdot f}{k}$, where $k$ is the propagation coefficient, $d$ is the distance from the transmitter and the receiver, $\beta$ is the attenuation coefficient which commonly ranges from 2 to 5 and $f$ represents the short-term (fast) fading components. In the case of Rayleigh fading, the r.v. $f$ is exponentially distributed with unit mean.

Let us define $L=k \cdot d^{\beta}$, which represents the averaged (with respect to fast fading) loss. Its version in logarithmic scale can be expressed as $L[d B]=k_{0}+k_{1} \ln d$, where $k_{0}=10 \log _{10} k$, $k_{1}=\beta \frac{10}{\ln 10}$.

Note that, for each air interface (intra-VAA and inter-VAA) we could have different propagation parameters $\left(k_{0}, k_{1}\right)$.

Being ancillary nodes distributed according to a PPP, the number of cooperating devices at a given node follows a Poisson distribution regardless of the model we consider to establish whether two nodes can communicate or not [8].

In this paper we assume that an ancillary node communicates with the main node (and viceversa) only if $L<L_{\text {th }}$, where $L_{\text {th }}$ represents the maximum loss tolerable by the communication system. The threshold $L_{\text {th }}$ depends on transmit power and receiver sensitivity. By assuming the propagation model stated above, the ancillary nodes communicate with the main node only if their distance is less than $d_{\mathrm{th}}=e^{\left(L_{\mathrm{th}}-k_{0}\right) / k_{1}}$. In particular, the mean number of cooperating nodes, denoted as $N$ hereafter, can be written as $\eta \pi d_{\mathrm{th}}^{2}$.

\section{ANALYSIS OF THE ERgodic CAPACITY FOR A V-MIMO SYSTEM}

\section{A. Capacity of a generic link}

In this section we focus on a generic hop (the $\ell$ th hop) of our scenario. We assume that this link is affected by $I^{(\ell)}$ co-channel interferers from other VAAs. We denote as $n_{\mathrm{R}}^{(\ell)}$ the number of nodes (cooperating and main) at the receiver VAA, and with $n_{\mathrm{T}_{\mathrm{k}}}^{(\ell)}$ with $k \in\left(0, \ldots, I^{(\ell)}\right)$, the number of transmit nodes of the desired $(k=0)$ and of the interfering $\left(k=1, \ldots, I^{(\ell)}\right)$ VAA. We denote as $n_{\mathrm{T}}^{(\ell)}=\sum_{k=0}^{I^{(\ell)}} n_{\mathrm{T}_{\mathrm{k}}}^{(\ell)}$, the total number of transmit nodes at the $\ell^{\text {th }}$ hop.

By considering the same assumptions made in [6], [7], the ergodic capacity in the $\ell^{\text {th }}$ hop in the case of uncorrelated 
Rayleigh fading is [7]

$$
\begin{aligned}
\bar{C}_{n_{\mathrm{T}}^{(\ell)}, n_{\mathrm{R}}^{(\ell)}, \mathbf{P}^{(\ell)}}^{(\ell)} & =\bar{C}\left(n_{\mathrm{T}}^{(\ell)}, n_{\mathrm{R}}^{(\ell)}, \tilde{\Psi}^{(\ell)}\right) \\
& -\bar{C}\left(\sum_{k=1}^{I^{(\ell)}} n_{\mathrm{T}_{\mathrm{k}}^{(\ell)}}^{(\ell)} n_{\mathrm{R}}^{(\ell)}, \Psi^{(\ell)}\right)
\end{aligned}
$$

where $\mathbf{P}^{(\ell)}=\left[P_{0}^{(\ell)}, P_{1}^{(\ell)}, \ldots, P_{I^{(\ell)}}^{(\ell)}\right], \quad$ being $\left(P_{0}^{(\ell)}, P_{1}^{(\ell)}, \ldots, P_{I^{(\ell)}}^{(\ell)}\right)$ the averaged (over fast fading) powers received by a given node of the $\ell^{\text {th }}$ hop, when transmitted by the desired user, the first and the $I^{\text {th }}$ interferer, respectively. $\bar{C}\left(n_{\mathrm{T}}, n_{\mathrm{R}}, \boldsymbol{\Theta}\right)$ is the ergodic capacity of a single link MIMO system with $n_{\mathrm{T}}$ transmit and $n_{\mathrm{R}}$ receive antennas, normalised with respect to the number of available resources. $\Theta$ is a diagonal matrix whose elements are the values of the power levels at the transmit antennas. In the presence of interference, $\Theta$ can be written as [7]:

$$
\mathbf{\Psi}^{(\ell)}=\frac{P_{1}^{(\ell)}}{\sigma_{\mathrm{N}}^{2}} \mathbf{I}_{n_{\mathrm{T}_{1}}} \oplus \frac{P_{2}^{(\ell)}}{\sigma_{\mathrm{N}}^{2}} \mathbf{I}_{n_{\mathrm{T}_{2}}} \oplus \cdots \oplus \frac{P_{I}^{(\ell)}}{\sigma_{\mathrm{N}}^{2}} \mathbf{I}_{n_{\mathrm{T}_{\mathrm{I}}}}
$$

and

$$
\tilde{\boldsymbol{\Psi}}^{(\ell)}=\frac{P_{0}^{(\ell)}}{\sigma_{\mathrm{N}}^{2}} \mathbf{I}_{n_{\mathrm{T}_{0}}} \oplus \boldsymbol{\Psi}^{(\ell)}
$$

where $\sigma_{\mathrm{N}}^{2}$ is the thermal noise power. Note that the eigenvalues of the matrices $\boldsymbol{\Psi}^{(\ell)}$ and $\tilde{\boldsymbol{\Psi}}^{(\ell)}$ have multiplicities larger than one.

Eqs. (1)-(3) show that the ergodic capacity analysis of VMIMO systems in the presence of multiple V-MIMO cochannel interferers requires the knowledge of the ergodic capacity $\bar{C}\left(n_{\mathrm{T}}, n_{\mathrm{R}}, \boldsymbol{\Theta}\right)$ of a MIMO system with $n_{\mathrm{T}}$ transmit and $n_{\mathrm{R}}$ receive antennas and with a covariance matrix $\Theta$ whose eigenvalues have arbitrary multiplicities.

We refer to [6] for the derivation of $\bar{C}\left(n_{\mathrm{T}}, n_{\mathrm{R}}, \boldsymbol{\Theta}\right)$ for brevity.

\section{B. Capacity between Source and Destination}

In the presence of a $Z$-hop communication protocol, the mean (with respect to fast fading fluctuations) capacity from the source to the destination, denoted by $\bar{C}_{\mathrm{S}, \mathrm{D}}$, is the minimum between the mean capacity in all hops; moreover, taking into account that each hop will use one out of the $R$ available resources [1], therefore the source-destination ergodic capacity can be written as

$$
\bar{C}_{\mathrm{S}, \mathrm{D}}=\min \left\{\frac{1}{R} \bar{C}_{n_{\mathrm{T}}^{(\ell)}, n_{\mathrm{R}}^{(\ell)}, \mathbf{P}^{(\ell)}}\right\}_{\ell=1, . ., Z}
$$

where $\bar{C}_{n_{\mathrm{T}}^{(\ell)}, n_{\mathrm{R}}^{(\ell)}}$ is given by (1).

We assume that the distance between two adjacent VAAs is $d$ meters, and that the contribution of an interfering VAA is significant only if its distance from the receiver VAA is less than $p \cdot d$, where $p$ is a suitable integer. This assumption implies that the maximum number of interfering VAAs, denoted as $I_{\max }^{(\ell)}$, is $2 p+1$, for $1<\ell<Z-2$. In particular, by setting $p=2$, we have: i) $\ell=1: I_{\max }^{(1)}=2$ with one interferer at distance $d$ and one interferer at distance $2 d$; ii) $1<\ell<Z-2$ : $I_{\max }^{(\ell)}=3$ with one interferer at distance $d$ and two interferers at distance $2 d$; iii) $\ell=Z-2: I_{\max }^{(Z-2)}=2$ with one interferer at distance $d$ and one interferer at distance $2 d$; iv) $\ell=Z-1$ and $\ell=Z: I_{\max }^{(Z-1)}=I_{\max }^{(Z)} 1$ with one interferer at distance $2 d$.

By assuming that all the nodes at the different VAAs transmit with the same power, $P_{\mathrm{T}}$, the averaged (over fast fading) power received by a given node of the receiver of the $\ell^{\underline{t h}}$ hop when transmitted by the desired user, $P_{0}^{(\ell)}$, will be equal to $P_{\mathrm{T}} k d^{-\beta}$. In the case of three interferers, we will have: $P_{1}^{(\ell)}=P_{3}^{(\ell)}=P_{\mathrm{T}} k(2 d)^{-\beta}$, being interferer 1 and 3 at distance $2 d$ from the receiver, and $P_{2}^{(\ell)}=P_{\mathrm{T}} k d^{-\beta}$, being interferer 2 at distance $d$. Since the values of the useful and the interfering received powers remain the same at the different hops, we will omit the apex ${ }^{(\ell)}$ in the following, and we will simply denote them as $P_{0}, P_{1}, P_{2}$ and $P_{3}$.

\section{OUTAGE PROBABILITY ANALYSIS}

Since the number of cooperating nodes is a r.v., there is a nonzero probability that the source-destination mean capacity, $\bar{C}_{\mathrm{S}, \mathrm{D}}$, is less than a given value, $C_{0}$, where $C_{0}$ depends on the specific application considered. In such scenario, a useful performance metric is the outage probability $P_{\text {out }} \triangleq \mathbb{P}\left\{\bar{C}_{\mathrm{S}, \mathrm{D}}<C_{0}\right\}$. We assume that slot assignments, VAA size and fading are independent at each hop. Under such assumptions, the outage events at each hop are independent and the outage probability at the $\ell^{\text {th }}$ hop, when $p=2$, can be written as

$$
\begin{aligned}
P_{\text {out }}^{(\ell)} & =\sum_{i, t_{0}, t_{1}, t_{2}, t_{3}, r} H\left(i, t_{0}, t_{1}, t_{2}, t_{3}, r, \mathbf{P}, C_{0}\right) \mathbb{P}\left\{I^{(\ell)}=i\right\} \\
& \times \mathbb{P}\left\{n_{\mathrm{T}_{0}}^{(\ell)}=t_{0}, n_{\mathrm{T}_{1}}^{(\ell)}=t_{1}, n_{\mathrm{T}_{2}}^{(\ell)}=t_{2}, n_{\mathrm{T}_{3}}^{(\ell)}=t_{3}, n_{\mathrm{R}}^{(\ell)}=r\right\}
\end{aligned}
$$

where

$$
\sum_{i, t_{0}, t_{1}, t_{2}, t_{3}, r} \triangleq \sum_{i=0}^{I_{\text {max }}^{(\ell)}} \sum_{t_{0}=1}^{M+1} \sum_{t_{1}=1}^{M+1} \sum_{t_{2}=1}^{M+1} \sum_{t_{3}=1}^{M+1} \sum_{r=1}^{M+1}
$$

and $H(\cdot)$ assumes the value of 1 when the capacity of the $\ell^{\text {th }}$ link, assuming the presence of $i$ interfering VAAs having $n_{\mathrm{T}_{1}}^{(\ell)}=t_{1}, n_{\mathrm{T}_{2}}^{(\ell)}=t_{2}$ and $n_{\mathrm{T}_{3}}^{(\ell)}=t_{3}$ transmitting nodes, $n_{\mathrm{T}_{0}}^{(\ell)}=$ $t_{0}$ nodes at the desired transmitter and $n_{\mathrm{R}}^{(\ell)}=r$ nodes at the receiver, is less than the threshold $C_{0} ; H(\cdot)$ is zero otherwise. Furthermore

$$
\begin{aligned}
& \mathbb{P}\left\{n_{\mathrm{T}_{0}}^{(\ell)}=t_{0}, n_{\mathrm{T}_{1}}^{(\ell)}=t_{1}, n_{\mathrm{T}_{2}}^{(\ell)}=t_{2}, n_{\mathrm{T}_{3}}^{(\ell)}=t_{3}, n_{\mathrm{R}}^{(\ell)}=r\right\} \\
& =Q\left(t_{0}, N\right)\left(\prod_{i=1}^{3} Q\left(t_{i}, N\right)\right) Q(r, N) .
\end{aligned}
$$

Since the number of cooperating nodes is limited, it is not distributed according to Poisson r.v., and has the following distribution:

$$
Q\left(t_{0}, N\right)= \begin{cases}P\left(t_{0}-1, N\right) & \text { for } t_{0}-1<M \\ 1-\sum_{l=0}^{M-1} P(l, N) & \text { for } t_{0}-1=M\end{cases}
$$


where $P(t, N) \triangleq \frac{N^{t}}{(t-1) !} e^{-N}$. Equivalent expressions can be written for $Q\left(t_{i}, N\right)$ for $i=1, . ., I_{\max }^{(\ell)}$ and $Q(r, N)$.

To evaluate the term $H\left(i, t_{0}, t_{1}, t_{2}, t_{3}, r, \mathbf{P}, C_{0}\right) \mathbb{P}\left\{I^{(\ell)}=i\right\}$ in (5), we have to distinguish between the different cases: $\ell=1,1<\ell<Z-2, \ell=Z-2, \ell=Z-1$ and $\ell=Z$. Now, let us focus on the case $1<\ell<Z-2$, which is characterized by the largest value of $I_{\max }^{(\ell)}$.

- $I^{(\ell)}=0$ :

$$
\begin{aligned}
& H\left(0, t_{0}, t_{1}, t_{2}, t_{3}, r, \mathbf{P}, C_{0}\right) \mathbb{P}\left\{I^{(\ell)}=0\right\} \\
& =\left(1-\frac{1}{R}\right)^{3} B\left(\frac{1}{R} \bar{C}_{t_{0}, r, \mathbf{P}}, C_{0}\right)
\end{aligned}
$$

where $\bar{C}_{t_{0}, r, \mathbf{P}}^{(\ell)}$ is given by (1), with $\mathbf{P}=\left[P_{0}\right], n_{\mathrm{T}}^{(\ell)}=t_{0}$, $n_{\mathrm{R}}^{(\ell)}=r$ and $\tilde{\boldsymbol{\Psi}}=\frac{P_{0}}{\sigma_{\mathrm{N}}^{2}} \mathbf{I}_{n_{\mathrm{T}_{0}}}$.

- $I^{(\ell)}=1$ :

$$
\begin{aligned}
& H\left(1, t_{0}, t_{1}, t_{2}, t_{3}, r, \mathbf{P}, C_{0}\right) \mathbb{P}\left\{I^{(\ell)}=1\right\} \\
& =\frac{1}{R}\left(1-\frac{1}{R}\right)^{2}\left[B\left(\frac{1}{R} \bar{C}_{t_{0}+t_{1}, r,\left[P_{0}, P_{1}\right]}, C_{0}\right)+\right. \\
& +B\left(\frac{1}{R} \bar{C}_{t_{0}+t_{2}, r,\left[P_{0}, P_{2}\right]}, C_{0}\right)+ \\
& \left.+B\left(\frac{1}{R} \bar{C}_{t_{0}+t_{3}, r,\left[P_{0}, P_{3}\right]}, C_{0}\right)\right]
\end{aligned}
$$

- $I^{(\ell)}=2$ :

$$
\begin{aligned}
& H\left(2, t_{0}, t_{1}, t_{2}, t_{3}, r, \mathbf{P}, C_{0}\right) \mathbb{P}\left\{I^{(\ell)}=2\right\} \\
& =\left(\frac{1}{R}\right)^{2}\left(1-\frac{1}{R}\right)\left[B\left(\frac{1}{R} \bar{C}_{t_{0}+t_{1}+t_{2}, r,\left[P_{0}, P_{1}, P_{2}\right]}, C_{0}\right)+\right. \\
& +B\left(\frac{1}{R} \bar{C}_{t_{0}+t_{1}+t_{3}, r,\left[P_{0}, P_{1}, P_{3}\right]}, C_{0}\right)+ \\
& \left.+B\left(\frac{1}{R} \bar{C}_{t_{0}+t_{2}+t_{3}, r,\left[P_{0}, P_{2}, P_{3}\right]}, C_{0}\right)\right] \\
& -I^{(\ell)}=3: \\
& \quad H\left(3, t_{0}, t_{1}, t_{2}, t_{3}, r, \mathbf{P}, C_{0}\right) \mathbb{P}\left\{I^{(\ell)}=3\right\} \\
& \quad=\left(\frac{1}{R}\right)^{3} B\left(\frac{1}{R} \bar{C}_{t_{0}+t_{1}+t_{2}+t_{3}, r,\left[P_{0}, P_{1}, P_{2}, P_{3}\right]}, C_{0}\right)
\end{aligned}
$$

Similar expressions can be obtained for the cases $\ell=1$, $Z-2, Z-1$ and $Z$, but they are not shown here for brevity.

Now, we introduce the complementary outage probability for the $\ell^{\text {th }}$ hop that is $P_{\text {in }}^{(\ell)} \triangleq 1-P_{\text {out }}^{(\ell)}$. Since $\bar{C}_{\mathrm{S}, \mathrm{D}}$ is proportional to the smallest capacity among the $Z$ links, the overall outage probability can be written as

$$
P_{\text {out }}=1-\left[P_{\text {in }}^{(1)}\right]^{2} \cdot\left[P_{\text {in }}^{(\ell)}\right]^{Z-4} \cdot\left[P_{\text {in }}^{(Z)}\right]^{2}
$$

where we took account of the fact that the cases $\ell=1$ and $\ell=Z-2$ have the same single-hop outage probability, such as the cases $\ell=Z-1$ and $\ell=Z$.

\section{Numerical Results}

In this section the behavior of the complementary outage probability for the $\ell^{\text {th }}$ hop, $P_{\text {in }}^{(\ell)}$, and the overall complementary outage probability, $P_{\text {in }} \triangleq 1-P_{\text {out }}$, is shown by varying different scenarios and system parameters. Results are obtained by setting, if not otherwise specified, $\eta=2 \cdot 10^{-2}$ $\left[m^{-2}\right], \sigma_{\mathrm{N}}^{2}=8 \cdot 10^{-15}[\mathrm{~W}]$ and $M=4$. We consider two different channel models for intra-VAA and inter-VAAs communication. In the first case, we set $k_{0}=41 \mathrm{~dB}, k_{1}=13.03$ $(\beta=3)$, and $L_{\mathrm{th}}=70 \mathrm{~dB}$; whereas we set $k_{0}=15 \mathrm{~dB}$ and we vary $\beta$ (i.e., $\left.k_{1}\right)$ for the inter-VAAs transmissions $\left(L_{\mathrm{th}}\right.$ is not fixed in this case, since we assume that the different VAAs are always connected).

Fig. 2 shows $P_{\text {in }}^{(\ell)}$ as a function of the requirement on the capacity, for different values of $R$, having set $\beta=3$ and $\rho=10[\mathrm{~dB}]$, being $\rho$ the SNR equal to $P_{0} / \sigma_{\mathrm{N}}^{2}$. All curves show a similar behavior, with $P_{\text {in }}^{(\ell)}$ monotonically decreasing. $P_{\text {in }}^{(\ell)}$ is larger for smaller $R$ because the SNR is low (10 $\mathrm{dB}$ ); therefore, smaller values of $R$ are beneficial in terms of augmented radio resource available, while the increased level of interference is not causing serious problems as the system tends to be noise-limited. On the opposite, when the SNR is larger (the curves are not shown for the sake of brevity), interference plays a more significant role with respect to noise; the advantage of a larger radio resource (when $R$ is small) is balanced by the disadvantage of an increased level of interference. Note that the number of transmit and receive antennas of the various hops is a discrete r.v. As a consequence, the source-destination capacity is a discrete r.v. too. This justifies the step-wise behavior of the curves.

Fig. 3 compares the performance obtained with Slotted Aloha to the case of a perfect centralised resource assignment, which makes the reuse more deterministic. As expected, performance levels tend to be more predictable in the latter case, and the level of capacity ensured becomes almost deterministic with $P_{\text {in }}^{(\ell)}\left(C_{0}\right)$ which takes a step-wise shape. The residual randomness in the performance is related to the random size of the VAAs. If the capacity requirement is very high, Slotted Aloha can provide better performance. However, for the values of outage probability of usual interest (i.e., $P_{\text {in }}^{(\ell)} \approx 0.9$ ), the centralised approach outperforms Slotted Aloha.

In Fig. 4 we show $P_{\text {in }}^{(\ell)}$ against $R$ for two values of the capacity requirement, in the case of Slotted Aloha, when $\rho=40 \mathrm{~dB}$ (this value has been chosen to make thermal noise negligible compared to interference). When $C_{0}$ is small the optimum value of $R$ is 2 ; on the other hand, when $C_{0}=10$ [bit/s/Hz], performance is optimised by choosing $R=5$. This optimum value is a trade-off between the need to reduce the impact of interference and to increase the amount of radio resource to be used. For smaller values of the SNR, the optimum $R$ tends to become smaller as expected: this is not shown here for the sake of conciseness.

Finally, in Fig. 5 we show the overall complementary outage probability against $Z$ for different values of $C_{0}$, having set $\rho=20 \mathrm{~dB}, R=2$ and $\beta=3$. The exact formula of the $P_{\text {in }}$ 


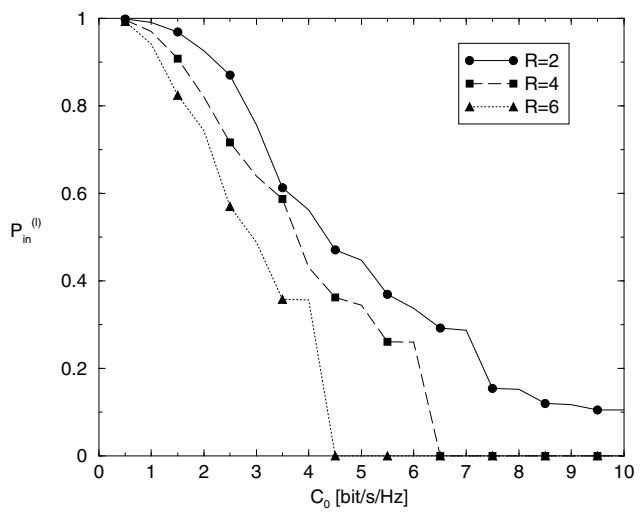

Fig. 2. $\quad P_{\mathrm{in}}^{(\ell)}$ as a function of $C_{0}$, when $\rho=10 \mathrm{~dB}$ and $\beta=3$.

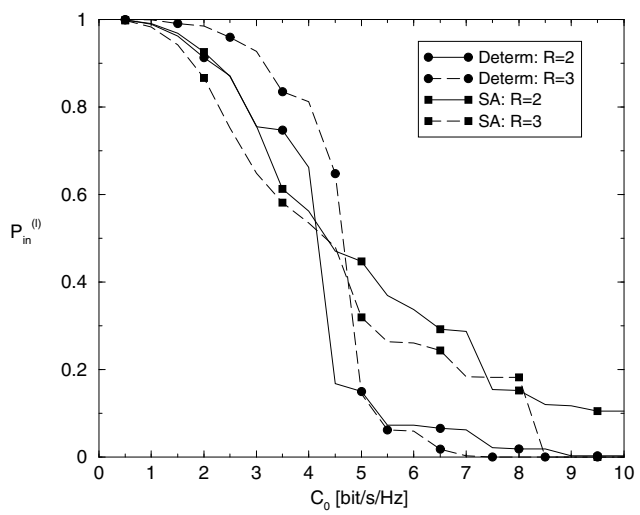

Fig. 3. $P_{\text {in }}^{(\ell)}$ as a function of $C_{0}$, in the case of slotted aloha and deterministic allocation of resources, when $\rho=10 \mathrm{~dB}$ and $\beta=3$.

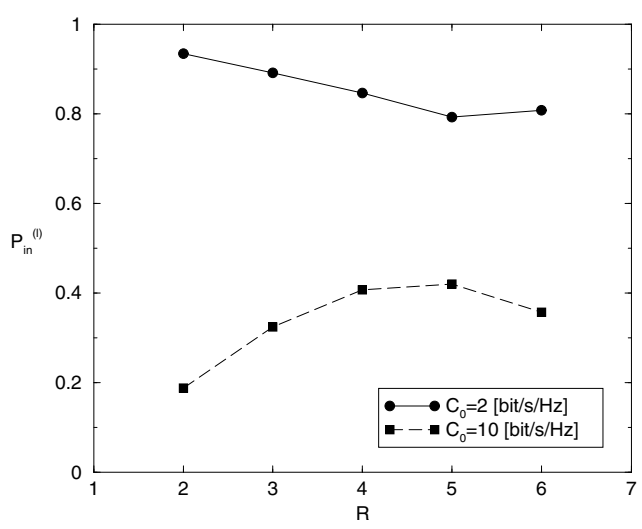

Fig. 4. $\quad P_{\text {in }}^{(\ell)}$ as a function of $R$ when $\rho=40 \mathrm{~dB}$ and $\beta=3$.

(given in Sec. IV) and a lower bound, given by $P_{\mathrm{in}, \mathrm{B}}=\left(P_{\mathrm{in}}^{(\ell)}\right)^{Z}$ (by considering the central link, i.e., $1<\ell<Z-2$ ), are considered. We can note that: (i) the complementary outage probability decreases when $Z$ gets larger; (ii) $P_{\text {in }}$ gets closer to the bound when augmenting $Z$ and/or decreasing $C_{0}$.

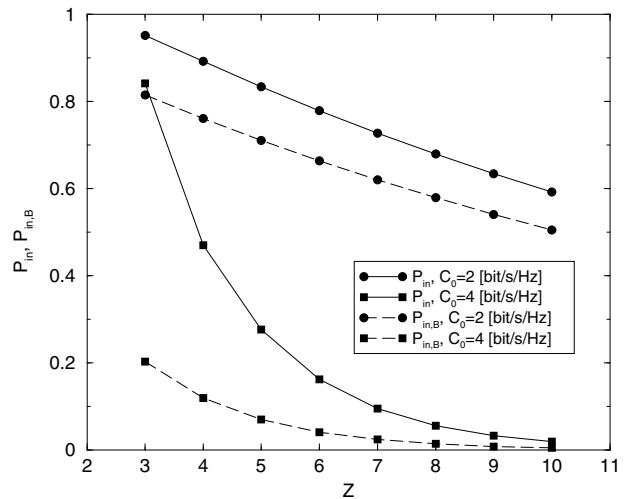

Fig. 5. $\quad P_{\text {in }}$ and $P_{\text {in,B }}$ as a function of $Z$ when $\rho=20 \mathrm{~dB}$ and $\beta=3$.

\section{CONCLUSION}

A multi-hop V-MIMO system, when Slotted Aloha is used to select the radio resource at each hop, has been investigated. Random location of cooperating nodes as well as propagation environment are considered and their effect on the overall performance is evaluated. Performance is computed in terms of outage probability, that is the probability that the capacity between source and destination is smaller than a given threshold. Results show that there exists an optimum value of $R$ that changes by varying the minimum capacity requested.

\section{ACKNOWLEDGMENT}

This work was supported by the European Commission in the framework of the FP7 Network of Excellence in Wireless Communications NEWCOM++ (contract n. 216715).

\section{REFERENCES}

[1] M. Dohler, A. Gkelias, H. Aghvami, "A resource allocation strategy for distributed MIMO multi-hop communication systems," IEEE Communications Letters, vol. 8, n. 2, pp. 99-101, Feb. 2004.

[2] H. Zhang and H. Dai, "On the capacity of distributed MIMO systems," in Proc. of the Conf. on Information Sciences and Systems (CISS 2004), Princeton University, Princeton, NJ, March 2004.

[3] A. del Coso, U. Spagnolini, C. Ibars, "Cooperative distributed MIMO channels in wireless sensor networks," IEEE Jornal on Sel. Areas Commun., vol. 25, n. 2, pp. 402414, Feb. 2007.

[4] W. Deng, X. Gao, "Three-hop cooperative diversity system and symbol error rate analysis," Int. Symp. on Intelligent Signal Processing and Communication Systems (ISPACS 2007), Nov. 28 2007-Dec. 12007.

[5] C. Buratti, A. Zanella,"Capacity analysis of two-hop virtual MIMO systems in a Poisson field of nodes," in Proc. of Veh. Tech. Conf. (IEEE VTC2009 - Spring), Barcelona, Spain, 26-29 April 2009.

[6] C. Buratti, A. Zanella, "The impact of channel reuse on performance of multi-hop virtual MIMO systems," in Proc. of IEEE ICUMT 2009, San Petersburg, Russia, Oct. 2009.

[7] M. Chiani, M. Z. Win, H. Shin, "Capacity of MIMO Systems in the Presence of Interference," in Proc. of IEEE Globecom 2006, Nov. 27 - Dec. 1, San Francisco, California, USA.

[8] J.F.C. Kingman, Poisson Processes, Oxford Univ. Press, 1993. 